\title{
A SHARP FORM OF THE AHLFORS' DISTORTION THEOREM, WITH APPLICATIONS
}

\author{
BY
}

D. H. HAMILTON

\begin{abstract}
The constant appearing in the asymptotic version of the Ahlfors' distortion theorem is 1 . Also it is shown that for mean l-valent functions $f=z+a_{2} z^{2} \ldots$ $\| a_{n+1}|-| a_{n}|| \leqslant 1$ for $n \geqslant N(f)$.
\end{abstract}

1. Introduction. The Ahlfors' distortion theorem [2] and its refinements are important in the theory of conformal mapping and its many applications. In this paper we obtain sharp bounds for the constants which arise in the theory of simultaneous growth and give some applications. First we state some definitions and indicate earlier work on these problems.

Let $u$ be a nonconstant harmonic function on a domain $\Omega$ in the complex sphere. Define the level set $l(c)=\{z \in \Omega \mid u(z)=c\},-\infty<c<\infty$, of $u$, and the quantity

$$
\theta(c)=\int_{l(c)}|* d u|
$$

where $\theta(c) \equiv 0$ if $l(c)=\phi$. For $-\infty \leqslant a<b \leqslant \infty, \Gamma(a, b)$ denotes the family $\{l(c) \mid a<c<b\}$, which will have module $\mu(a, b)$ that satisfies

$$
\mu(a, b)=\int_{a}^{b} \frac{d c}{\theta(c)}
$$

provided $(a, b) \subset u(\Omega)$. For subsets $\Omega_{j} \subset \Omega$ we similarly define $\theta_{j}(c), \mu_{j}(a, b)$ etc.

Now let $S$ be an infinite horizontal strip of thickness $\pi$ and $S_{j}, k$ disjoint infinite horizontal substrip of thickness equal to $\pi / k$. Then Jenkins and Oikawa [8, p. 60] define the maximum simultaneous growth of $u$ in $k$ directions to mean there are sequences $\left\{b_{n}\right\}$ and $\left\{\xi_{n}+i \eta_{n, j}\right\} \subset S_{j}$ such that

$$
\begin{gathered}
{[a, \infty) \subset \bigcap_{j=1}^{k} u\left(S_{j}\right), \quad \xi_{n} \rightarrow \infty, \quad a<b_{n} \leqslant u\left(\xi_{n}+i \eta_{n j}\right),} \\
\lim _{n \rightarrow \infty}\left(\mu\left(a, b_{n}\right)-\frac{\xi_{n}}{\pi k}\right)>-\infty .
\end{gathered}
$$

They prove that as $\xi+i \eta$ approaches $\infty$ in any compact substrip of $S_{j}$, there is a uniform limit

$$
\lim \left\{\frac{\mu_{j}(a, u(\xi+i \eta))}{k}-\frac{\xi}{\pi}\right\}=\alpha_{j}, \quad-\infty<\alpha<\infty .
$$

Received by the editors December 21, 1982 and, in revised form, May 27, 1983.

1980 Mathematics Subject Classification. Primary 30C55. 
These results of Jenkins and Oikawa elegantly generalize earlier results of Eke [4] for functions $f(z)$ which are areally mean $p$-valent on the unit disk. In this case $u=\log |f(z)|$ and $\theta(\log R) \equiv p(R)$ measures the mean $p$-valence of $f(z)$. Eke [4, p. 149] finds that $f(z)$ has maximal growth at $k$ distinct points $e^{i \phi_{1}}, \ldots, e^{i \phi_{k}}$ if and only if $z_{j}$ are points "near" the rays $\arg (z)=\phi_{j}$ which satisfy $R=\left|f\left(z_{j}\right)\right|$ so that as $R \rightarrow \infty$,

$$
\prod_{j=1}^{k} R\left(1-\left|z_{j}\right|\right)^{-2 p / k}
$$

tends to a nonzero finite limit $\alpha$.

This result is not unlike a distortion theorem of Aharanov and Srebro [1]. Suppose that $D$ is locally $k$ connected at $\infty$, i.e., $D \cap\{|w|>R\}$ has exactly $k$ components for large $R$. The canonical example is the domain $\Omega$ where $\partial \Omega$ is $k$ equally separated rays (not necessarily of equal length). In both cases assume that 0 is an interior point of $D$ and $\Omega$, and there is a function $f(z)=z+\cdots$ which maps $\Omega$ onto $D$ such that $\lim _{|z| \rightarrow \infty}|f(z)|=\infty$. Now let $z_{j}$ be points in the $j$ th segment of $D$ such that $\left|f\left(z_{j}\right)\right|=R$; then Aharanov and Srebro prove

$$
\prod_{j=1}^{k} \frac{R}{\left|z_{j}\right|}<16 .
$$

The constant 16 is best possible, i.e., for every $\varepsilon>0$ there are $f$ and $R$ such that the product is more than $16-\varepsilon$. However 16 is never attained, furthermore as $R \rightarrow \infty$ it is found that the bound is substantially smaller than 16 .

We shall give precise bounds for the constants arising in the above results. For $n=1$ this has already been done. Jenkins and Oikawa introduce normalization

$$
u(\xi+i \eta)=u_{0}+\lambda \xi+o(1)
$$

as $\xi \rightarrow-\infty$, and the reduced module

$$
\tilde{\mu}(b)=a / \theta(a)+\mu(a, b)
$$

under the assumption $(-\infty, \infty) \subset u(\Omega)$ and prove

$$
\lim \left(\tilde{\mu}(u(\xi+i \eta))-\frac{1}{\pi} \xi\right)=\alpha \leqslant \frac{u_{0}}{2 \pi \lambda}
$$

uniformly as $\xi+i \eta \rightarrow+\infty$ in a substrip of S. Hayman [7] and Eke [4] had shown that if $f(z)=z^{p}+\cdots$ is mean $p$-valent in the unit disk then as $|z| \rightarrow \infty$

$$
\lim \frac{(1-|z|)^{2 p}}{|z|^{p}}|f(z)|=\alpha \leqslant 1 .
$$

2. Statement of results. For functions $u$ harmonic on the strip $S$ we say that $u$ has maximal growth in $k$ directions if in each substrip $S_{j}$ thickness $\pi / k$

$$
\begin{gathered}
\mu_{j}(a, b)<\infty, \quad-\infty<a<b<\infty, \\
(-\infty, \infty) \subset u\left(S_{j}\right), \\
\overline{\lim } u(\xi+i \eta)=+\infty,
\end{gathered}
$$


as $\xi+i \eta \rightarrow+\infty$ in some proper substrip of $S_{j}$. Then we prove

THEOREM 1. Suppose that $u(\xi+i \eta)$ has maximal simultaneous growth in $k$ directions. Also suppose that $u(\xi+i \eta)=u_{0}+\lambda \xi+o(1)(\lambda>0)$ uniformly as $\xi \rightarrow-\infty$. Then if $\xi_{j}+i \eta_{j} \rightarrow \infty$ uniformly in each proper substrip of $S_{j}, b=u\left(\xi_{j}+i \eta_{j}\right)$, we have

$$
\varlimsup\left(\tilde{\mu}(b)-\frac{1}{k \pi} \sum_{j=1}^{k} \xi_{j}(b)\right)=\alpha,
$$

where $-\infty \leqslant \alpha \leqslant u_{0} / 2 \pi \lambda$ with equality only for $u=u_{0}+\lambda \xi$. Furthermore if $\alpha>-\infty$ then, for each $j, \tilde{\mu}_{j}(b) / k-\xi_{j}(b) / \pi \rightarrow \alpha_{j}$, where $-\infty<\alpha_{j}$ and $\sum_{j=1}^{k} \alpha_{j} \leqslant u_{0} /(2 \pi \lambda)$.

COROLlaRY 1. Suppose that $f(z)=z^{p}+\cdots$ is areally mean p-valent in $\{|z|<1\}$ and satisfies

$$
\varlimsup_{z \rightarrow e^{i \phi_{j}}}|f(z)|=\infty
$$

in some Stoltz angle at $e^{i \phi_{j}} ; e^{i \phi_{1}}, \ldots, e^{i \phi_{k}}$ distinct points. Then if $z_{j}$ are points such that $R=\left|f\left(z_{j}\right)\right| \rightarrow \infty$ we have

$$
\varlimsup \prod_{j=1}^{k} R\left(1-\left|z_{j}\right|\right)^{2 p / k} \rightarrow \alpha
$$

uniformly for $z_{j} \rightarrow e^{i \phi_{j}}$ in a Stoltz angle. Furthermore,

$$
\alpha \leqslant \prod_{j \neq q}\left|e^{i \phi_{j}}-e^{i \phi_{q}}\right|^{-2 p / k}
$$

with equality only for

$$
f(z)=\frac{z^{p}}{\left(1-z e^{-i \phi_{1}}\right)^{2 p / k} \cdots\left(1-z e^{-i \phi_{k}}\right)^{2 p / k}} .
$$

REMARK. If $f(z)$ is univalent then the Golusin inequality shows that the product is bounded (see Pommerenke [10]). For general $p$-valent functions, Lucas [9] shows that the product is uniformly bounded.

CoROllary 2. Suppose that $f(z)=z+\cdots$ maps a $k$-star $\Omega$ onto a domain $D$ which is locally $k$-connected at $\infty$. Then if $z_{j}$ is a point in the jth segment of $\Omega$ such that as $\left|f\left(z_{j}\right)\right|=R \rightarrow \infty$ as $z_{j} \rightarrow \infty$,

$$
\varlimsup \prod_{j=1}^{k} \frac{R}{\left|z_{j}\right|}=\alpha \leqslant 1
$$

with equality only for $f(z)=z$.

REMARK. There is no Stoltz angle conditions in the above theorem.

The case $n=2, p=1$ in Corollary 1 is particularly interesting. The work of Hayman [6], Eke [4] and Hamilton [5] show that if

$$
f(z)=z+a_{2} z^{2}+\cdots
$$


is univalent in $\{|z|<1\}$ then

$$
\varlimsup|| a_{n+1}|-| a_{n}|| \leqslant 1 .
$$

It was conjectured that 1 is attained only for $f(z)=z\left(1-z e^{-i \phi_{1}}\right)^{-1}\left(1-z e^{-i \phi_{2}}\right)^{-1}$, the problem being to find which univalent functions $f$ in the Eke class with maximal growth at $e^{i \phi_{1}}, e^{i \phi_{2}}$ satisfy

$$
\varlimsup_{n \rightarrow \infty} \min _{j=1,2} n^{-1}\left|f\left(\left(1-n^{-1}\right) e^{i \phi_{1}}\right)\right|=\left|e^{i \phi_{1}}-e^{i \phi_{2}}\right|^{-1} .
$$

Corollary 1 immediately gives this. In fact, we prove

THEOREM 2. Suppose that $f(z)=z+a_{2} z^{2}+\cdots$ is areally mean 1-valent in $\{|z|<1\}$. Then for $n>N(f),\left\|a_{n+1}|-| a_{n}\right\| \leqslant 1$. Equality is attained if and only if $f(z)=z /\left(1-z e^{-i \phi_{1}}\right)\left(1-z e^{-i \phi_{2}}\right)$, and $\left(\phi_{1}-\phi_{2}\right) / \pi$ is rational.

3. Proof of Theorem 1. The following result is similar to results of Eke [3, p. 108] and Jenkins and Oikawa [8, p. 44].

LEMMA 1. If $u(\xi+i \eta)$ harmonic on $\left\{\xi_{0}<\xi,|\eta|<\pi / 2\right\}$ satisfies

$$
\mu(a, b)<\infty(-\infty<a<b<\infty)
$$

and

$$
\widetilde{\lim } u(\xi+i \eta)=\infty
$$

as $\xi+i \eta \rightarrow \infty$ for $|\eta|<\pi / 2-\delta_{0}$ (some $\delta_{0}>0$ ) then as $\xi \rightarrow \infty,|\eta|<\pi / 2-\delta$ $(\delta>0)$, the uniform limit

$$
\lim (\mu(a, u(\xi+i \eta))-\xi / \pi)=\alpha, \quad-\infty \leqslant \alpha<\infty,
$$

exists.

We note that (11) implies $\varlimsup \lim u\left(\xi+i \eta_{0}\right)=\infty$ for some $\eta_{0}$. Furthermore, we may assume $\mu(a, \infty)=\infty$, otherwise (12) is obvious, with $\alpha=-\infty$. These two comments are almost the hypothesis of Theorem 2 [8, p. 44] and the proof is essentially the same. For every $c$, let $\sigma(c)$ be the component of

$$
\{\xi+i \eta \in S: u(\xi+i \eta)<c\}
$$

containing $\xi_{0}$. Similar to Lemma 2 [8, p. 45], we prove that there is an $a_{0}$ such that, for every $c>a_{0}, \sigma(c)$ is bounded above, and thus the unbounded component $\tau(c)$ of $S-\sigma(c)$ is determined uniquely. Then set

$$
\Gamma(c)=S \cap \overline{\sigma(c)} \cap \overline{\tau(c)}
$$

which is an arc in $l(c)$ which joins the upper and lower edges of the strip. Also define

$$
\xi^{\prime}(c)=\inf \{\xi: \xi+i \eta \in \Gamma(c)\}
$$

and

$$
\xi^{\prime \prime}(c)=\sup \{\xi: \xi+i \eta \in \Gamma(c)\},
$$

then it is known that $\lim _{c \rightarrow \infty} \xi^{\prime}(c)=\infty$. 


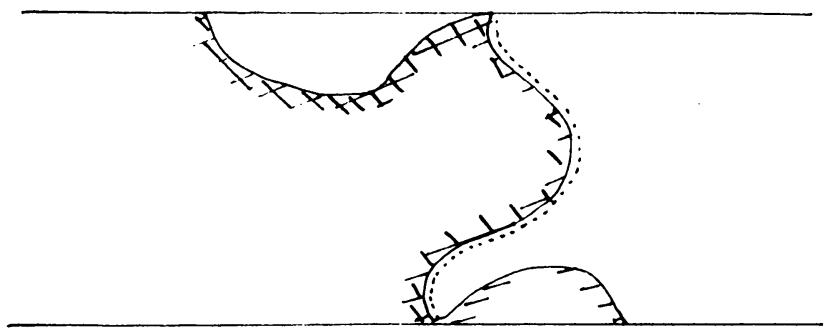

I) $u(z)<c$

II $\sigma(c)$

$\cdots \Gamma(c)$

Define a function $\rho(t)=t^{3} / 9\left(1+t^{2} / 3\right)$, then $[8$, p. 46]

$$
\mu(a, b) \leqslant \frac{\xi^{\prime \prime}(b)-\xi^{\prime}(a)}{\pi}-\rho\left(\xi^{\prime \prime}(c)-\xi^{\prime}(c)\right)
$$

for $a<c<b, l(a), l(b), l(c)$ joining the upper and lower edge of $S$.

One should note that $\alpha$ depends on $a$. It is shown that if $\lim \left(\mu\left(a_{0}, b\right)-\xi^{\prime}(b) / \pi\right)$ $=\alpha_{0}$ then $\alpha=\alpha_{0}+\mu\left(a, a_{0}\right)$. In the case $\alpha_{0}>-\infty, \lim _{c \rightarrow x} \overline{\xi^{\prime \prime}}(c)-\xi^{\prime}(c)=0$.

We now apply these results to the strips $S_{j}$ of Theorem 1 . Let $S_{j}(\delta)$ be an infinite horizontal strip of thickness $\pi / k-\delta$ such that $\overline{S_{j}(\delta)} \subset S_{j}$. Finally we need the inequality

$$
\mu(a, b) \leqslant \frac{1}{k^{2}} \sum_{j=1}^{k} \mu_{j}(a, b)
$$

$[8$, p. 40]. Now we may assume

$$
\varlimsup\left(\mu\left(a_{0}, b_{n}\right)-\frac{1}{\pi k} \sum_{j=1}^{k} \xi_{j n}\right)>-\infty
$$

for some sequence $\xi_{j n}+i \eta_{j n} \in S_{j}(\delta), b_{n}=u\left(\xi_{j n}+i \eta_{j n}\right)$ tends to $\infty$. By (14) we obtain

$$
\varlimsup\left(\sum_{j=1}^{k}\left(\frac{1}{k} \mu_{j}\left(a_{0}, b_{n}\right)-\frac{\xi_{j n}}{\pi}\right)\right)>-\infty
$$

as $n \rightarrow \infty$. Now each summand, by Lemma 1, tends to a number $\alpha_{j}<\infty$ and, in particular, is bounded above. Thus we see that (15) implies that each $\alpha_{j}>-\infty$.

Now choose $a<c$ near $-\infty, b>a_{0}$ so that $l(a), l(c), l(b)$ join the upper and lower edges of each substrip $S_{j}$. Then as $\alpha_{j}>-\infty$, by Lemma 1 ,

$$
\frac{1}{k} \mu_{j}(a, b)+o(1) \geqslant \alpha_{j}+\frac{\xi_{j}^{\prime \prime}}{\pi}(b) \geqslant \alpha_{j}+\frac{\xi_{j}^{\prime}}{\pi}(c)+\frac{\mu_{j}(c, b)}{k}
$$

by (13). Also if $c \rightarrow-\infty$ more slowly than $a$ we find, that as $u(\xi+i \eta)=u_{0}+\lambda \xi+$ $o(1), \xi \rightarrow-\infty$,

$$
\mu_{j}(a, c)=\int_{a}^{c} \frac{d c}{\theta_{j}(c)}=\frac{(c-a) k}{\pi \lambda}+o(1)
$$


Thus (16) is bounded below by

$$
\alpha_{j}+\frac{\xi_{j}^{\prime}(c)}{\pi}+\frac{\mu_{j}(a, b)}{k}-\frac{(c-a)}{\pi \lambda}+o(1)
$$

Consequently,

$$
\frac{(c-a)}{\pi \lambda} \geqslant \alpha_{j}+\frac{\xi_{j}^{\prime}(c)}{\pi}+o(1) .
$$

Now âs $c \rightarrow-\infty, \xi_{j}^{\prime}(c)=\left(c-u_{0}\right) / \lambda+o(1)$ and therefore as $a \rightarrow-\infty$ we obtain

$$
\alpha_{j} \leqslant-\left(a-u_{0}\right) / \pi \lambda \text {. }
$$

We now complete the proof of Theorem 1. As $b \rightarrow \infty$ from $\alpha_{j}>-\infty$ we have

$$
\begin{aligned}
\sum_{j=1}^{k} \frac{\xi_{j}(b)}{\pi} & =\sum_{j=1}^{k}\left(\frac{\mu_{j}(a, b)}{k}-\alpha_{j}+o(1)\right) \\
& \geqslant k \mu(a, b)+\frac{k}{\pi \lambda}\left(a-u_{0}\right)+o(1)
\end{aligned}
$$

as $a \rightarrow-\infty$, using (14) and (17). Thus

$$
-\frac{1}{\pi \lambda}\left(a-u_{0}\right) \geqslant \mu(a, b)-\frac{1}{\pi k} \sum_{j=1}^{k} \xi_{j}(b)+o(1) .
$$

Now

$$
\tilde{\mu}(b)-\frac{1}{\pi k} \sum_{j=1}^{k} \xi_{j}(b)=\frac{a}{\pi \lambda}+\left(\mu(a, b)-\frac{1}{\pi k} \sum_{j=1}^{k} \xi_{j}(b)\right) .
$$

Thus

$$
\frac{u_{0}}{\pi \lambda} \geqslant \varlimsup\left(\tilde{u}(b)-\frac{1}{\pi k} \sum_{j=1}^{k} \xi_{j}(b)\right) .
$$

Furthermore, by (14) we see that there is equality if and only if $\xi^{\prime}(c)=\xi^{\prime \prime}(c)$ for $a<c<b$. Thus the level curves are vertical and hence $u(\xi+i \eta)=u_{0}+\lambda \xi$.

Proof of Corollary 1. We map the unit disk onto the $w$-plane by

$$
w=\frac{z}{\left(1-z e^{-i \phi_{1}}\right)^{2 / k} \cdots\left(1-z e^{-i \phi_{k}}\right)^{2 / k}},
$$

so that $w(|z|<1)$ is the complex plane cut along $k$ rays separated by angle $2 \pi / k$. This domain is then mapped onto an infinite horizontal strip by

$$
\xi+i \eta=\frac{1}{2} \log (w)
$$

Suppose that $f(z)$ is areally mean $p$-valent, i.e.

$$
\int_{0}^{R} R p(R) d R \leqslant \frac{1}{2} p R^{2}
$$

for $0<R<\infty$, where $p(R)$ is the proportion of the circle $|w|=R$ covered by $f(|z|<1)$, counting multiplicity. Furthermore if $f(z)=z^{p}+\cdots$, and we define 
$f(\xi+i \eta)=\log |f(z)|$ we see that

$$
u(\xi+i \eta)=2 p \xi+o(1)
$$

near $-\infty$. Also

$$
\mu\left(\log R_{1}, \log R_{2}\right)=\frac{1}{2 \pi} \int_{R_{1}}^{R_{2}} \frac{d R}{R p(R)} .
$$

Now if $\xi+i \eta=w(z)$, then as $z \rightarrow e^{i \phi_{j}}$,

$$
\xi=-\frac{1}{k} \log (1-|z|)-\log \beta_{j},
$$

where $\beta_{j}=\prod_{j \neq q}\left|e^{i \phi_{q}}-e^{i \phi_{j}}\right|^{1 / k}$. Consequently, if $z_{j} \rightarrow e^{i \phi_{j}}, \xi_{j}+i \eta_{j}=w\left(z_{j}\right),\left|f\left(z_{j}\right)\right|$ $=R$,

$$
k \log R+\frac{2 p}{k} \sum_{j=1}^{k} \log \left(1-\left|z_{j}\right|\right)=k\left(\log R+\frac{2 p}{k} \sum_{j=1}^{k}\left(-\xi_{j}-\log \beta_{j}\right)\right) .
$$

It is known for mean $p$-valent functions [4, p. 164] that

$$
\int_{R_{1}}^{R_{2}} \frac{d R}{R p(R)} \geqslant \frac{1}{p} \log \frac{R_{2}}{R_{1}} .
$$

Consequently by (19) and (21) $\log R \leqslant 2 \pi p \tilde{\mu}(\log R)$. Thus by (20)

$$
k \log R+\frac{2 p}{k} \sum_{j=1}^{k} \log \left(1-\left|z_{j}\right|\right) \leqslant 2 \pi p k\left(\tilde{\mu}(\log R)-\frac{1}{\pi k} \sum_{j=1}^{k}\left(\xi_{j}+\log \beta_{j}\right)\right) .
$$

Therefore by Theorem 1

$$
\varlimsup\left(k \log R+\frac{2 p}{k} \sum_{j=1}^{k} \log \left(1-\left|z_{j}\right|\right)\right) \leqslant-2 p \sum_{j=1}^{k} \log \beta_{j}
$$

as $u_{0}=0$ with equality only for $u=2 p \xi$. This completes the proof of Corollary 1 .

Proof of CoRollary 2. The proof of a weaker version of this result with convergence in Stoltz angles is just a special case of Corollary 1. The condition that $D$ is locally $k$-connected at $\infty$ allows us to remove these restrictions. For then if $R$ is large, $D \cap\{|w| \geqslant R\}$ has exactly $k$ components. Consequently in each strip $S_{j}$, $l_{j}(\log R)=\Gamma_{j}(R)$. Thus

$$
\begin{aligned}
& \xi_{j}^{\prime}(\log R)=\inf \left\{\xi: \xi+i \eta \in l_{j}(\log R)\right\}, \\
& \xi_{j}^{\prime \prime}(\log R)=\sup \left\{\xi: \xi+i \eta \in l_{j}(\log R)\right\}
\end{aligned}
$$

and our previous analysis then may be applied to yield the result.

Proof of Theorem 2. If $f(z)$ has maximal growth at one point then Hayman [6, p. 243] shows that

$$
|| a_{n+1}|-| a_{n} \| \rightarrow \alpha \leqslant 1
$$

with equality only for $f(z)=z /\left(1-z e^{-i \phi_{1}}\right)^{2}$. If $f(z)$ does not have maximal growth at one or two points then Eke [4, p. 151] proves that

$$
\left|a_{n+1}\right|-\left|a_{n}\right| \rightarrow 0 \text {. }
$$


Hamilton [5] gives his proof of

$$
\varlimsup|| a_{n+1}|-| a_{n}|| \leqslant 1
$$

for univalent functions with maximal growth at two points $e^{i \phi_{1}}, e^{i \phi_{2}}$, however most of his method also works for mean 1-valent functions. Set

$$
\alpha_{j}(n)=n^{-1} f\left(\left(1-\frac{1}{n}\right) e^{-i \phi_{i}}\right) .
$$

Then Eke [4, p. 185] proves that

$$
a_{m}=\alpha_{1} e^{-i m \phi_{1}}+\alpha_{2} e^{-i m \phi_{2}}+o\left\{\max \left(\left|\alpha_{1}\right|,\left|\alpha_{2}\right|\right)\right\}
$$

for $m=n, n+1$ as $n \rightarrow \infty$.

Hamilton considers sequences $n_{k}$ according to whether

$$
\varlimsup_{k} \max \left(\left|\alpha_{1}\left(n_{k}\right)\right|,\left|\alpha_{2}\left(n_{k}\right)\right|\right)<\infty
$$

or not. In the first case, by (24),

$$
\left|a_{n+1}-e^{i \phi_{1}} a_{n}\right|=\alpha_{2}\left(e^{-i(n+1) \phi_{2}}-e^{-i n \phi_{2}+\phi_{1}}\right)+o(1)
$$

if $\alpha_{1}=\max \left(\left|\alpha_{1}\right|,\left|\alpha_{2}\right|\right)$. Hence as $n_{k} \rightarrow \infty$

$$
|| a_{n+1}|-| a_{n} \| \leqslant\left|\alpha_{2}\right|\left|e^{i \phi_{1}}-e^{i \phi_{2}}\right|+o(1) .
$$

Now from Corollary 1 we have

$$
\left|\boldsymbol{\alpha}_{2}\right| \leqslant\left|e^{i \phi_{1}}-e^{i \phi_{2}}\right|^{-1}+o(1)
$$

with asymptotic equality only for $z /\left(1-z e^{-i \phi_{1}}\right)\left(1-z e^{-i \phi_{2}}\right)$. This proves the theorem for a subsequence satisfying (25). For the other case, $\lim \max \left(\left|\alpha_{1}\right|,\left|\alpha_{2}\right|\right)=\infty$. In this case we prove, as $n_{k} \rightarrow \infty$,

$$
\left|a_{n+1}\right|-\left|a_{n}\right| \rightarrow 0 \text {. }
$$

The proof is exactly the same as Hamilton's (and similar to Eke's proof of (23)). This completes the proof of Theorem 2 .

\section{REFERENCES}

1. D. Aharanov and U. Srebro, Boundary behaviour of conformal and quasiconformal mappings, Ann. Acad. Sci. Fenn. Ser. A I Math. 8 (1982), 3-42.

2. L. Ahlfors, Untersuchungen zur Theorie der konformen Abbildung und der gonzen Functionen, Acta Soc. Sci. Fenn. Nova Ser. A 1 (1930), no. 9.

3. B. G. Eke, Remarks on Ahlfors' distortion theorem, J. Analyse Math. 19 (1967), 97-134.

4. The asymptotic behaviour of areally mean p-valent functions, J. Analyse Math. 20 (1967), $147-212$.

5. D. H. Hamilton, The successive coefficients of univalent functions, J. London Math. Soc. (2) 25 (1982), 122-128.

6. W. K. Hayman, On the successive coefficients of univalent functions, J. London Math. Soc. (2) 38 (1963), 228-243.

7.

8. J. A. Jenkins and K. Oikawa, On the growth of slowly increasing unbounded harmonic functions, Acta Math. 124 (1970), 37-63.

9. K. W. Lucas, A two point bound for areally mean p-valent functions, J. London Math. Soc. (2) 43 (1968), 487-494.

10. Ch. Pommerenke, On the Hankel determinants of univalent functions, Mathematika 14 (1967), $108-112$. 